\title{
Postratamiento en escala piloto del lixiviado del relleno sanitario Antanas (Pasto-Nariño) por filtración-adsorción con arena, antracita y carbón activado
}

Pilot Scale Posttreatment of Leachate from the Antanas (Pasto-Nariño) Sanitary Landfill by Filtration Adsorption with Sand, Anthracite and Activated Carbon

\begin{abstract}
Andrés Felipe López Guerrero ${ }^{(1)}$, Iván Andrés Sánchez Ortiz ${ }^{(2)}$
(1) M.Sc. en Ingeniería Sanitaria y Ambiental, Universidad del Valle, Cali, Colombia. Jefe de Medio Ambiente Planta Papeles del Cauca SA Colombiana Kimberly Colpapel. andres.felipe.lopez@correounivalle.edu.co.

(2) M.Sc. M.Sc. en Ingeniería Civil, UNESP, Brasil y Ph.D. (c) en Ingeniería Civil, Universidade Federal de Viscosa, Brasil. Profesor Asociado Departamento Recursos Hidrobiológicos, Universidad de Nariño. Pasto, Colombia. Grupo de Investigación en Acuicultura (GIAC), Universidad de Nariño. iaso@udenar.edu.co
\end{abstract}

Recibido 4 de Agosto de 2015. Modificado 15 de octubre de 2015. Aprobado 27 octubre de 2015.

DOI: http//:dx.doi.org/10.16924/riua.v0i43.840

\begin{abstract}
Palabras clave
Carbón activado, lixiviado, residuos sólidos, tratamiento.

\section{Resumen}

Esta investigación evaluó el postratamiento del lixiviado del relleno sanitario Antanas mediante tres filtros ascendentes en serie con arena, antracita y carbón activado granular. Se evaluaron rangos de tasas de filtración de 20-35, $60-100$ y $150-300 \mathrm{~m}^{3} / \mathrm{m}^{2 *} \mathrm{~d}$ correspondientes a tiempos de contacto con el carbón de 48, 24 y 8 minutos. El seguimiento a cada condición se efectuó durante un mes, las remociones medias de DQO para dichas tasas y tiempos fueron 60,2, 54,3 y 31,4\% respectivamente. El mejor desempeño del sistema dependió fundamentalmente del aumento del tiempo de contacto y no de la reducción de las tasas de filtración.
\end{abstract}

\section{Key words}

Activated Carbon, Leachate, Treatment, Solid Waste

\section{Abstract}

This study assessed the posttreatment of leachate from the Antanas landfill by means three upflow filters with sand, anthracite and granular activated carbon. Filtration rates with ranges of $20-35,60-100$ and $150-300 \mathrm{~m}^{3} / \mathrm{m}^{2 *} \mathrm{~d}$, corresponding to carbon contact times of 48, 24 and 8 minutes were assessed. Each condition was monitored for a month, and the average COD removal values for the rates were $60.2,54.3$ and $31.4 \%$ respectively. The best system performance depended mainly on increased contact time and not on the reduction of filtration rates.

\section{INTRODUCCIÓN}

La tasa promedio de generación de residuos sólidos - RSmunicipales para países en vías de desarrollo es del orden de $0,60 \mathrm{~kg} / \mathrm{hab} . d$ y en naciones desarrolladas, de 2,13 kg/ hab.d (The World Bank, 2012). Según Wilson, Velis \& Rodic (2013), el manejo y disposición de los residuos está entre las dimensiones principales del manejo integrado y sostenible de los residuos municipales. Adicionalmente, dichos autores manifiestan que un relleno sanitario ingenierilmente diseñado, y adecuadamente operado y controlado, es parte esencial de cualquier sistema moderno de manejo de residuos.
La densificación poblacional y la urbanización de las regiones generan alto crecimiento en la cantidad de RS producidos en las naciones. En el caso de Colombia, los valores estimados de producción diaria de RS -en ton/d- es de 19.000 en el 2000 (Suárez, 2000), 22.808 en el 2002 (OPS, 2002) y 25.079 para 2008 (Noguera \& Olivero, 2010). Aunque los rellenos sanitarios presentan grandes ventajas, la formación continua de lixiviados altamente contaminantes, de composición variable y flujo cambiante, supone un problema ambiental muy serio y, según Noguera \& Olivero (2010), su tratamiento inadecuado representa uno de los principales problemas 
operativos en el contexto colombiano. De acuerdo con Lopes et al. (2015), en los rellenos sanitarios, los residuos sólidos domiciliarios están sometidos a procesos de descomposición química y biológica, que dan origen a efluentes líquidos y gaseosos; la fracción líquida está formada por la humedad contenida en los residuos, producto del proceso de biodegradación anaerobia y por el aporte de las precipitaciones y otros tipos de infiltración que percolan al relleno.

Dado que el lixiviado es un efluente de difícil tratamiento y de composición compleja, generalmente para obtener resultados que atiendan los límites ambientales para vertimiento, se hace necesaria una secuencia de procesos de tratamiento, cada uno de ellos actuando sobre determinado(s) componente(s) del lixiviado (Lopes et al., 2015). Cuando el tratamiento biológico es insuficiente para depurar el líquido, se puede requerir una barrera adicional de proceso; por ello, algunos autores proponen como alternativa el postratamiento por procesos físico-químicos, para obtener altas eficiencias de remoción (Kurniawan, Wai-Hung \& Chan, 2006). De acuerdo con Kawahigashi et al. (2014), cuando los sistemas de tratamiento basados en procesos biológicos y físicoquímicos convencionales aun no son capaces de remover la demanda química de oxígeno —DQO — recalcitrante y otros contaminantes hasta los niveles exigidos por las normas de vertimiento, o de adecuar su calidad de tal manera que no causen impactos negativos al medio ambiente, se hace necesaria la investigación de técnicas o procesos alternativos y complementarios para el postratamiento de dichos lixiviados.

El relleno sanitario Antanas — RSA —, localizado en el municipio de Pasto, se encarga de la disposición final de los RS de la capital del departamento de Nariño y de 30 municipios aledaños. El área total disponible del RSA es de 100 ha, en las que se ha previsto la disposición de los residuos en tres vasos de la siguiente manera: el Vaso I, ya clausurado y con una extensión de 5 ha, operó desde marzo de 2001 hasta junio de 2009; el Vaso II, actualmente en operación, en el cual se estima realizar la disposición de residuos hasta el 2027, posee una superficie de 7 ha; el Vaso III con un área para disposición de RS de 8 ha, donde se proyecta una capacidad de operación aproximada hasta el 2041 (López, Zambrano \& Safa, 2013).

El sistema actual de tratamiento de los lixiviados del RSA - cuyas unidades constitutivas y tren de tratamiento serán descritos en el siguiente ítem-, recibe el líquido proveniente del Vaso II (lixiviado nuevo) y del Vaso I (lixiviado maduro), los cuales generan caudales y concentraciones variables de lixiviado como resultado de la incidencia de las precipitaciones en la zona de disposición final de los residuos. Pese a que tal condición genera variabilidad en la eficiencia del tratamiento para remoción de contaminantes del lixiviado, según López, Zambrano \& Safa (2013), el RSA entre 2010 y 2012 cumplió con lo estipulado en la normatividad ambiental pues, el lixiviado tratado removió más del $80 \%$ de la carga de sólidos suspendidos (en promedio 89\%) y de demanda bioquímica de oxígeno - DBO- (en promedio 97\%). Sin embargo, dicha variabilidad, sumada a la previsión de un volumen creciente de residuos a ser dispuestos y de líquido a tratar, sugiere la necesidad de evaluar opciones de tratamiento adicionales que permitan mantener la calidad del efluente final lo más uniforme posible y dentro de los límites definidos por la legislación.

El objetivo de la presente investigación fue evaluar el postratamiento de lixiviado del RSA por medio de un sistema conformado por tres unidades de filtración: el primer y segundo filtro, con fines de tratamiento físico para retención de sólidos, tuvieron como medio de soporte arena negra y antracita respectivamente; el tercer filtro, con carbón activado granular - CAG - fue implementado para ejercer las labores de columna de adsorción. Para la operación del sistema se determinaron tres tasas de filtración en medio granular y tres tiempos de contacto - TC - con el CAG. El desempeño de las unidades de tratamiento fue monitoreado a lo largo de un mes para cada tasa de filtración y TC evaluados. Para ello, se determinó la eficiencia en la remoción de DQO y color aparente, así como el comportamiento del pH y la temperatura.

\section{Materiales Y MÉTODOS}

\section{CARACTERÍSTICAS DEL RSA, RESIDUOS SÓLIDOS, ESQUEMA DE TRATAMIENTO EXISTENTE Y LIXIVIADO}

La investigación se desarrolló con el lixiviado efluente de la laguna de maduración del RSA de la ciudad de Pasto, ubicado en las coordenadas N: 633.103, E: 979.569 y a una altitud de $2750 \mathrm{msnm}$. El relleno sanitario está ubicado a $13 \mathrm{~km}$ de la ciudad, registra una precipitación media de 1300 mm/año y una temperatura promedio de $12{ }^{\circ} \mathrm{C}$.

El origen de los lixiviados tratados y la secuencia del sistema de tratamiento del RSA actualmente en operación son: el lixiviado que se produce en el Vaso I -en el que se dispusieron aproximadamente 657.000 toneladas de RS- es conducido por gravedad hacia un reactor UASB y su efluente es bombeado mediante un sistema con caudal de diseño de 2 L/s hacia una laguna de igualación en la zona del Vaso II -en operación, que para el 2012 recibió en promedio 294,3 ton/d de RS-, donde se juntan los lixiviados provenientes de los dos vasos. El lixiviado combinado posteriormente pasa hacia una laguna aireada donde se suministra oxígeno mediante un aireador superficial de $15 \mathrm{HP}$; su efluente pasa hacia un reactor de lodos activados y posteriormente hacia unidades de tratamiento físico químico (procesos de coagulación, floculación, sedimentación) y finalmente hacia una laguna de maduración, cuyo tiempo de retención hidráulica es de aproximadamente un mes. El líquido tratado es conducido por tubería hasta el punto de descarga final, en el cruce del río Bermúdez y la quebrada Robles, ubicado a 10,2 km del RSA. El sistema de conducción combina tramos por flujo a presión y flujo libre. 
De acuerdo con una caracterización física de los residuos sólidos del municipio de Pasto, realizada en el 2007, la distribución media de los componentes de los RS fue la siguiente: residuos de comida putrescible, 70\%; papel y cartón, 8,31\%; plástico, 8,57\%; textiles, 1,41\%; caucho, 0,92\%; maderas, $0,73 \%$; podas, $0,39 \%$; vidrio, $3,92 \%$; metales, $1,51 \%$; baterías, $0,44 \%$; otros, 3,80\%; con contenido de humedad medio del 72,56\% y de material orgánico del 44,15\% (Alcaldía de San Juan de Pasto, 2007). El mismo documento afirmó que el sistema de tratamiento de los lixiviados presenta un desempeño variable, y que la eficiencia media de remoción de la DBO5 y la DQO es del 79\%. Adicionalmente, se presentó una caracterización del lixiviado generado en el RSA, cuyos resultados resaltan los valores de los siguientes parámetros: color, 3600 UPC; turbiedad, 927 NTU; sólidos totales, 9250 mg/L; sólidos suspendidos, $990 \mathrm{mg} / \mathrm{L}$; grasas y aceites, 73,5 mg/L; pH, 7,21 unidades; nitritos, 0,28 mg/L; nitratos, 59,5 mg/L; DBO5, 3493 mg/L; DQO, 8217 mg/L; dureza total 1285 mg/L; sulfatos, 950 mg/L; amonio, 308 mg/L.

\section{SISTEMA DE TRATAMIENTO PILOTO EVALUADO}

Durante la investigación se realizaron pruebas a escala piloto del postratamiento del efluente de la planta de tratamiento de lixiviados del RSA por medio de un sistema conformado por tres unidades de filtración. En el sistema no se controlaron las variables medioambientales ni las cargas contaminantes afluentes, las cuales estuvieron relacionadas con las condiciones hidrológicas y climáticas presentes durante el desarrollo de la investigación.

La sección transversal de las unidades de filtración estuvo definida por tubería sanitaria comercial en PVC de 6"; la primera columna tuvo como medio filtrante arena negra silícea, la segunda tuvo antracita y la tercera CAG, que actuó como columna de adsorción. Cada lecho granular estuvo soportado por una capa de grava de $5 \mathrm{~cm}$ de espesor; los medios filtrantes propiamente dichos ocuparon en cada columna cerca del $60 \%$ del volumen efectivo del filtro, representando una altura de medio granular de $1,80 \mathrm{~m}$. A cada material se le realizó análisis granulométrico siguiendo el procedimiento descrito en la Norma Técnica Colombiana NTC 1522 (ICONTEC, 1999). A partir de esto, se determinó para cada uno de ellos el tamaño efectivo $\left(\mathrm{D}_{10}\right)$ y el coeficiente de uniformidad $\left(C U=D_{60} / D_{10}\right)$ que, según Crites \& Tchobanoglous (1998), son las características principales de los medios filtrantes utilizados para tratamiento de aguas residuales. Para realizar el retrolavado de las unidades se implementó un sistema de llaves que permitió intervenir cada unidad por separado. Para ello, inicialmente se impidió el paso del afluente y se dejó drenar el filtro; para el lavado por flujo ascensional por 3 minutos se utilizó agua potable a una tasa de $400-500 \mathrm{~m}^{3}$ / $\mathrm{m}^{2 *} \mathrm{~d}$ y se retornaron los filtros y las columnas a normal funcionamiento.

\section{Parámetros EVALUAdos Y DiseÑo EXPERIMENTAL}

Se realizó el seguimiento del sistema para 3 tasas de filtración y 3 tiempos de contacto durante un mes para cada tasa de filtración y TC evaluados. Para el análisis de los datos se planteó un diseño factorial con un arreglo en bloques completamente al azar, con 2 factores y 3 niveles, donde la variable de respuesta fue la concentración (eficiencia de remoción) de la DQO. Los factores analizados y sus niveles fueron: Factor A, unidades de tratamiento: Filtro 1 (arena), Filtro 2 (antracita), Filtro 3 (columna de adsorción con CAG); Factor B rangos de caudales para tres tasas de filtración que definieron las fases de evaluación del desempeño del sistema de postratamiento: Fase 1: 20-35 $\mathrm{m}^{3} / \mathrm{m}^{2 *} \mathrm{~d}$, Fase 2: $60-100 \mathrm{~m}^{3} / \mathrm{m}^{2 *} \mathrm{~d}$, Fase 3: $150-300 \mathrm{~m}^{3} / \mathrm{m}^{2 *} \mathrm{~d}$. Los tiempos de contacto medios adoptados para el sistema de adsorción por CAG, correspondientes con las tasas de filtración, fueron de 48, 24 y 8 minutos.

Mediante un análisis de contrastes multivariados con un nivel de confianza del 95\% se verificó el modelo del diseño experimental empleado y se aplicó la prueba de esfericidad de Mauchly para medidas repetidas en donde se obtuvieron diferencias significativas. Las eficiencias de remoción medias, obtenidas a partir de las concentraciones de DQO afluentes y efluentes para cada nivel de los factores evaluados, fueron comparadas para determinar la tasa de filtración y TC que presentaron mejor desempeño en términos de remoción de la DQO; el cálculo de la eficiencia global de remoción se realizó de forma multiplicativa, con base en las fracciones remanentes, según lo recomendado por von Sperling (2012).

La evaluación del desempeño de cada nivel de los factores se realizó a lo largo de un mes; se midieron tres días a la semana, durante 4 horas consecutivas los parámetros in situ: caudal, temperatura, $\mathrm{pH}$ y color aparente, para un total de 48 muestras semanales, totalizando 192 muestras mensuales por columna. Para la DQO se tomó una muestra cada hora durante 4 horas consecutivas para un total de 16 muestras semanales por punto de muestreo y de 48 muestras por cada tasa de filtración y TC evaluados. La medición de los parámetros físico químicos evaluados se realizó siguiendo los procedimientos establecidos por Rice et al. (2005) según los siguientes métodos: temperatura, método termo-eléctrico (2550 B); pH, método electrométrico (4500-H B); color aparente, método espectrofotométrico (2120 C); DQO, método por reflujo cerrado (5220D). En la Figura 1 se presenta un esquema del sistema evaluado con los diferentes puntos de muestreo. Al finalizar cada periodo de seguimiento se retiraron los medios granulares y fueron reemplazados por nuevos materiales.

\section{Resultados}

Con base en la caracterización granulométrica de los medios utilizados en las columnas de filtración y adsorción 


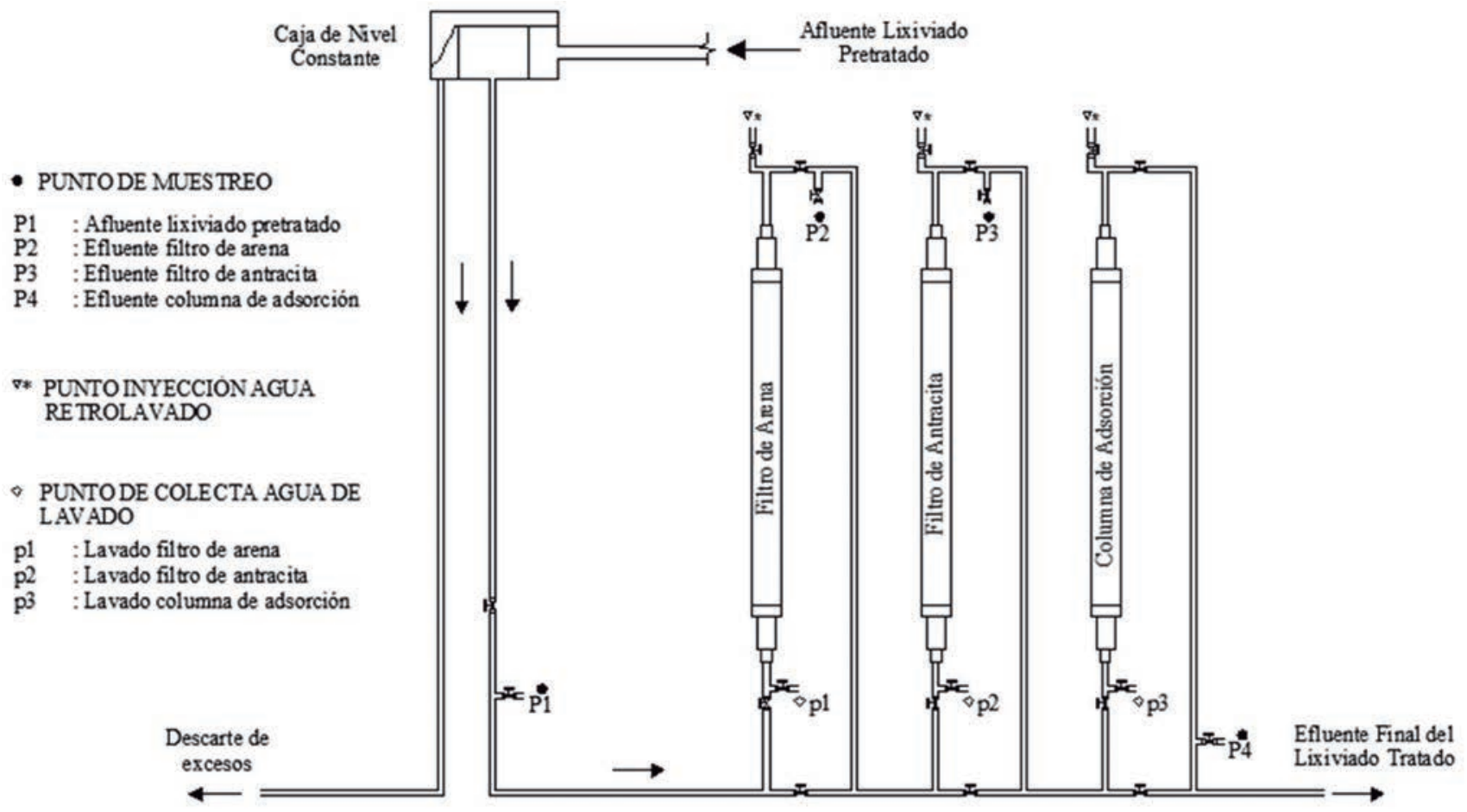

Figura 1. Vista frontal sistema de columnas de filtración y adsorción evaluado Fuente: Elaboración propia, 2015.

se determinó que los tamaños efectivos de la arena negra, la antracita y el CAG fueron respectivamente de 0,15, 0,42 y $0,61 \mathrm{~mm}$. Con los datos anteriores y los valores correspondientes de $\mathrm{D}_{30}$ y $\mathrm{D}_{60}$ se determinó que los $\mathrm{CU}$ de tales medios fueron respectivamente de 6,40, 3,33, 1,72, lo que evidenció una alta uniformidad en el carbón activado.

Los caudales medidos a lo largo de las tres fases ejecutadas fueron de 10,8 \pm 1,78 mL/s, 20,1 $\pm 2,45 \mathrm{~mL} / \mathrm{s}$ y 34,5 \pm $2,81 \mathrm{~mL} / \mathrm{s}$, los cuales se encontraron dentro de los rangos de tasas de filtración y tiempos de contacto estipulados para la evaluación del sistema de postratamiento de los lixiviados.
En los experimentos se registraron temperaturas medias del lixiviado tratado afluente de 16,4 $\pm 1,0,17,3 \pm 2,8$ y $17,7 \pm 1,4^{\circ} \mathrm{C}$. En los tres ensayos se evidenció un incremento en la temperatura del efluente de la columna de adsorción con respecto a la del afluente. Los valores medios de dicho incremento fueron de $1,89,1,1$ y $0,8^{\circ} \mathrm{C}$ para los tiempos de contacto de 48, 24 y 8 minutos respectivamente. Este comportamiento pudo deberse al hecho de que la adsorción suele ser un proceso exotérmico.

A continuación, en la Tabla 1 se presentan los valores medios calculados para $\mathrm{pH}$ y color aparente -en unidades de $\mathrm{pH}$

\begin{tabular}{c|c|c|c|c|c|}
\hline Parámetro & Fase & Afluente bruto & $\begin{array}{c}\text { Efluente filtro } \\
\text { de arena }\end{array}$ & $\begin{array}{c}\text { Efluente filtro } \\
\text { de antracita }\end{array}$ & $\begin{array}{c}\text { Efluente filtro } \\
\text { de CAG }\end{array}$ \\
\hline \multirow{2}{*}{ pH } & Primera & 8,20 & 8,00 & 7,80 & 7,50 \\
\cline { 2 - 6 } & Segunda & 8,02 & 7,98 & 8,01 & 7,85 \\
\cline { 2 - 6 } & Tercera & 7,79 & 7,64 & 7,71 & 7,30 \\
\hline \multirow{2}{*}{ Color Aparente } & Primera & 309,3 & 287,0 & 288,7 & 95,0 \\
\cline { 2 - 6 } & Aparente & 3157,6 & 2813,5 & 2657,3 & 1723,6 \\
\cline { 2 - 6 } & Tercera & 1572,8 & 1669,4 & 1727,2 & 1269,2 \\
\hline
\end{tabular}

Tabla 1. Valores medios marginales de remoción de la DQO

Fuente: Elaboración propia, 2015. 
y platino de color, respectivamente- en los puntos de muestreo (afluente y efluente de las unidades de pos-tratamiento) durante las tres fases de la investigación.

En general, se registró un ligero descenso en el pH del lixiviado al ser sometido a los tres ensayos del tratamiento de filtración-adsorción, sin justificación aparente. Cabe resaltar que al inicio de cada una de las fases el carbón activado y los medios filtrantes fueron reemplazados, con el objetivo de mantener condiciones similares para cada etapa del experimento.

En las tres fases se registraron bajas remociones de color aparente por parte de las dos primeras columnas granulares, y una mayor eficiencia en la columna de adsorción, cuyas eficiencias medias en las fases 1,2 y 3 superaron respectivamente el 65, 45 y 29\%. Ello pudo deberse a la baja presencia de material en suspensión y predominio de sólidos disueltos en el lixiviado, asociados a la materia orgánica refractaria, la cual junto con el color verdadero han sido efectivamente removidos en postratamiento de lixiviados por adsorción, como lo han reportado Kawahigashi et al. (2014).

Las concentraciones medias de la DQO afluente en las fases 1, 2 y 3 del experimento fueron de 265, 2030 y 1715 $\mathrm{mg} / \mathrm{L}$, todas ellas menores a la concentración media reportada por la Alcaldía de San Juan de Pasto (2007), probablemente por la creciente presencia de lixiviado maduro; y las concentraciones del efluente final fueron de 99, 788 y 1002 $\mathrm{mg} / \mathrm{L}$. En la Figura 2 se presentan las eficiencias medias marginales de remoción por parte de las unidades de tratamiento para cada tasa de aplicación. En ella se puede apreciar que la columna de adsorción asumió de manera mayoritaria la remoción de la DQO, cuyas eficiencias medias calculadas con relación al efluente del filtro de antracita en las fases 1, 2 y 3 fueron respectivamente de 60,2, 43,7 y 23,6\%. Tales eficiencias son comparables a las reportadas por Kawahigashi et al. (2014), quienes evaluaron el postratamiento de los lixiviados de la ciudad de Rolândia, en el estado de Paraná-Brasil, inicialmente sometidos a stripping de amoníaco, seguido de tratamiento biológico por lodos activados y posteriormente por coagulación, floculación y sedimentación, así como por adsorción mediante carbón activado granular. En dicha experiencia, los autores registraron eficiencias de remoción entre 45 y 76\% de la DQO por medio de la adsorción con diferentes tipos de carbón activado, para un tiempo de contacto de 20 minutos. Dado el importante efecto del aumento del tiempo de contacto evidenciado en esta investigación, es recomendable efectuar experimentos con otros tiempos de tal manera que se logren conjugar y optimizar los criterios relativos a la eficiencia del tratamiento y los costos de implementación de las unidades para adsorción.

Los resultados indicaron que el desempeño global del sistema de postratamiento mejoró con el aumento del tiempo de contacto, mas no necesariamente con la reducción de las tasas de aplicación en los medios filtrantes. Esto sugiere que en el lixiviado tratado del RSA, la materia orgánica se encontró principalmente en la forma disuelta y no en la forma particulada, que justifica las mayores remociones obtenidas mediante la adsorción, situación que ha sido comentada por Sanjay, Amit \& Mukherjee (2013). En términos prácticos, esto se traduce en la obtención de buenos resultados mediante tiempos de contacto de 48 minutos con tasas de aplicación altas, del orden de $150-300 \mathrm{~m}^{3} / \mathrm{m}^{2 *}$ d. Lo anterior propiciaría economía de área y volumen en los dos primeros filtros, los cuales asumen un trabajo físico de protección de la columna de contacto contra colmatación.

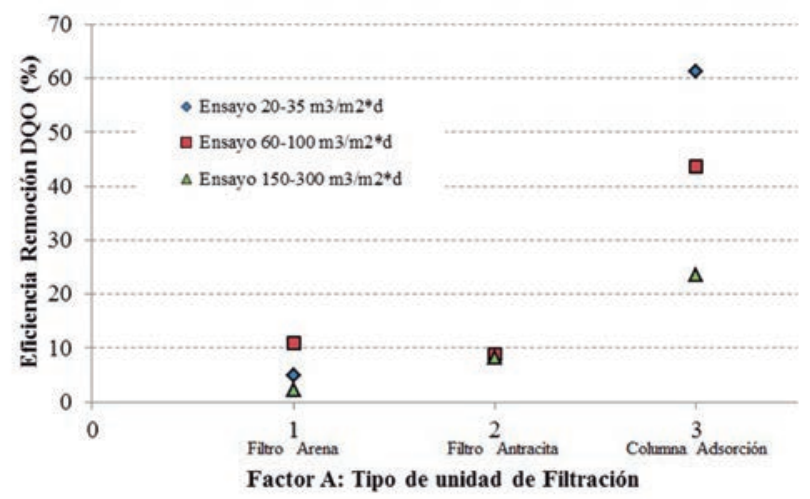

Figura 2. Medias marginales de remoción de la DQO Fuente: Elaboración propia, 2015.

Como ya fue comentado, y según se puede apreciar en la Figura 2, la adsorción fue fundamental para la remoción de la DQO, cuyo valor global obtenido por el sistema de postratamiento superó el 61\% para los mayores tiempos de contacto evaluados. En ese sentido, Kurniawan \& Wai-Hung (2009) encontraron remociones de DQO de 82\% combinando la adsorción en CAG con oxidación química utilizando H2O2; y, cuando utilizaron únicamente la oxidación química con peróxido de hidrógeno las remociones fueron de 33\%. Así mismo, Kurniawan et al., (2006) determinaron remociones de 86\% en DQO combinando ozonización con adsorción sobre CAG, y remociones del 35\% evaluando únicamente la ozonización. Esto ratifica la eficiencia del carbón activado en la remoción de materia orgánica.

\section{Conclusiones}

El sistema evaluado es una alternativa válida para el postratamiento de lixiviados. Su buen desempeño se fundamenta principalmente en la remoción de la materia orgánica disuelta por medio de la adsorción en carbón activado granular.

El desempeño global del sistema de postratamiento mejora con el aumento del tiempo de contacto y no necesariamente con la reducción de las tasas de aplicación en los medios filtrantes, por ello, es factible aplicar la tecnología con bajas 
áreas y volúmenes en las dos primeras unidades del sistema, que protegen de colmatación prematura a la columna de contacto.

\section{REFERENCIAS}

Alcaldía de San Juan de Pasto. (2007). Plan de gestión integral de residuos sólidos, PGIRS 2007-2022. San Juan de Pasto, Alcaldía Municipal.

Crites, R. \& Tchobanoglous, G. (1998). Small and descentralized wastewater management systems. First edition. Boston: McGraw Hill.

Instituto Colombiano de Normas Tecnicas y Certificación -ICONTEC. (1999). Norma técnica colombiana NTC 1522, Ensayo para determinar la granulometría por tamizado. Bogotá: ICONTEC.

Kawahigashi, F., Mendes, M.B., Assunção, V. G., Gomes, V. H., Fernandes, F., Hirooka, E. Y. \& Kuroda, E. K. (2014). Pós-tratamento de lixiviado de aterro sanitário com carvão ativado. Eng. Sanit. Ambient., 19(3), 235-244. doi: 10.1590/S1413-41522014019000000652

Kurniawan, T. A., Wai-Hung, L. \& Chan, Y.S.G. (2006). Physico-chemical treatments for removal of recalcitrant contaminants from landfill leachate. Journal of Hazardous Materials, 129, 80-100. doi:10.1016/j.jhazmat.2005.08.010

Kurniawan, T. A. \& Wai-Hung. (2009). Removal of refractory compounds from stabilized landfill leachate using an integrated $\mathrm{H} 2 \mathrm{O} 2$ oxidation and granular activated carbon (GAC) adsorption treatment. Water Research, 43, 40794091. doi: 10.1016/j.watres.2009.06.060

Lopes, C. M., Reis, L. S., Santos, T. A. \& Fernandes, F. (2015). Sequência de tratamentos para lixiviado de aterro sanitário utilizando os processos físico-químico convencional e Fenton como pós-tratamento. Revista DAE, 199, 47-57. doi: http://dx.doi.org/10.4322/dae.2014.145

López, A., Zambrano, J. \& Safa, El-L. (2013). Tratamiento de lixiviados y su efecto en la calidad del agua. En Publicaciones UNIMAR (Ed.), Agua para la vida: Memorias de la IV Semana Nacional de la Ciencia, la Tecnología y la Innovación, Nariño 2012 (pp. 137-156). San Juan de Pasto: Editorial Publicaciones UNIMAR, Universidad Mariana.

Modin, H., Persson, K. M., Andersson, A. \& Van Praaghc, M. (2011). Removal of metals from landfill leachate by sorption to activated carbon, bone meal and iron fines. Journal of Hazardous Materials, 189, 749-754. doi: 10.1016/j.jhazmat.2011.03.001

Morawe, B., Ramteke, D.S. \& Vogelpohl, A. (1995). Activated carbon column performance studies of biologically treated landfill leachate. Chem. Eng. Process. 34, 299303. doi:10.1016/0255-2701(94)04017-6

Noguera, K. M. \& Olivero, J. T. (2010). Los rellenos sanitarios en Latinoamérica: caso colombiano. Rev. Acad. Colomb. Cienc., 34(132), 347-356.

Organización Panamericana de la Salud-OPS. (2002). Evaluación regional de los servicios de manejo de residuos sólidos municipales, informe analítico de Colombia levaluación 2002. Washington: OPS.

Rice, E.W., Baird, R.B., Eaton, A.D. \& Clesceri, L.S. (Eds). (2005). Standard Methods for the Examination of Water and Wastewater, $21^{\circ}$ ed. Washington. D.C.: American Public Health Association (APHA), American Water Works Association (AWWA) Water Environment Federation (WEF)

Sanjay, M., Amit, D. \& Mukherjee, S. (2013). Applications of adsorption process for treatment of landfill leachate. Journal of Environmental Research And Development, 8(2), 365-370.

Shrawan, K.S., Timothy, G.., Mazyck, D. \& Boyer, H.T. (2012). Equilibrium and intra-particle diffusion of stabilized landfill leachate onto micro- and meso-porous activated carbon. Water Research, 46, 491-499. doi:10.1016/j. watres.2011.11.007

Suárez, G.C.I. (2000). Problemática y gestión de residuos sólidos peligrosos en Colombia. Innovar, 15, 41-52.

The World Bank. (2012). What A Waste: A global review of solid waste management, Urban Development Series. Washington, DC.: Urban Development \& Local Government Unit World Bank.

von Sperling, M. (2012). Introducción la calidad del agua y al tratamiento de aguas residuales. Pasto, Colombia: Editorial Universitaria Universidad de Nariño.

Wilson, D. C., Velis, C. A. \& Rodic, L. (2013). Integrated sustainable waste management in developing countries. Waste and Resource Management, 166, 52-68. doi: 10.1680/warm.12.00005 